\title{
RELACIONES ENTRE AGUA Y SEDIMENTO EN RIOS DE ORIGEN TORRENCIAL
}

\author{
E. Ruiz, A. Echeandía*, F. Romero \\ Departamento de Ingeniería Química y del Medio Ambiente. E.T.S.I. Industriales y de Telecomunicación. \\ Universidad del País Vasco. BILBAO. \\ * S.I.M.A. Servicio de Investigación y Mejora Agraria. Departamento de Agricultura y Pesca. \\ Gobierno Vasco. DERIO, Bizkaia.
}

Palabras clave: relación agua-sedimento, régimen torrencial.

\begin{abstract}
WATER-SEDIMENT RELATIONS IN TORRENTIAL RIVERS FROM THE BASQUE COUNTRY.

This paper includes composition and main parameters of waters and sediments from three torrential rivers of the Basque Country (NE of Spain), namely Bidasoa, Oria and Lea.

Waters are mainly classified through major constituens both by Piper's unitary triangular diagrams and by their aggressive or incrustant character versus calcium carbonate. Most of studied samples are rich in calcium and bicarbonates, with $\mathrm{pH}$ values close to carbonic equilibrium. The average compositions of sediments are different for studied basins, with a high calcium content in the case of sediments from Oria river.

It seems that the compositions of sediments is mainly determined by materials from rock disintegration because equilibrium situations with water have not yet been achieved. Finally an estimation of mineralogical composition of sediments is proposed.
\end{abstract}

\section{INTRODUCCIÓN}

Existen muchas dificultades en el estudio de ecosistemas acuáticos tanto por su complejidad como por los cambios multifactoriales en el flujo y química del agua. Las crecidas y los cambios en la gestion del río pueden drásticamente afectar a la configuración del lecho del mismo, así como a la distribución del sedimento, plantas y fauna. Estos cambios causan problemas en el establecimiento de modelos aplicados a la hidroquímica de corrientes naturales.

No obstante, cambios estacionales de algunas especies químicas disueltas en corrientes calcáreas han sido adscritas a ciclos de producción primaria (CASEY \& NEWTON, 1973; CASEY, 1976). Utilizando canales artificiales de circulación construídos para simular flujo estable e input químico uniforme se han realizado estudios que permiten relaciones entre los procesos hidrológicos, químicos y biológicos en corrientes calcáreas.

Los procesos naturales y los cambios inducidos por el hombre producen variaciones en el $\mathrm{pH}$ del agua, salinidad y condiciones redox, que pueden provocar movilización de especies químicas acumuladas en los sedimentos. En ríos o corrientes de tipo torrencial los fenómenos de erosión, transporte y sedimentación son factores que van a determinar el comportamiento del sistema.

El transporte de sedimentos está fuertemente relacionado con la hidrología y la geomorfología de la cuenca. Las variaciones que se producen son espaciales y temporales. La variación espacial viene marcada, sobre todo, por la naturaleza y el tamaño del sedimento, ya que los tamaños gruesos (grava y arena) tendrán menor movilidad que los finos (limos y arcillas) (SALOMONS \& FORSTNER, 1984) que son transportados como carga suspendida (EINSTEIN \& KRONE, 1962).

Las variaciones temporales estarán marcadas principalmente por la climatología y el régimen de los ríos. Los ríos de características torrenciales arrastran cantidades grandes de sedimentos por lo que se establecen variaciones en la composición de sus aguas (FORSTNER \& PROSSI, 1978).

En este trabajo se pretenden describir las relaciones en- 
tre la composición de las aguas y los sedimentos de ríos de carácter torrencial.

\section{ÁREA DE ESTUDIO}

Los ríos objeto de estudio son Bidasoa, Oria y Lea, situados en la Comunidad Autónoma Vasca y Navarra, y pertenecientes a la cuenca hidrográfica del Norte de España (Fig. 1). Los tres ríos desembocan en el mar Cantábrico.

El río Bidasoa tiene su cuenca repartida entre Navarra y Guipúzcoa. Recorre 50 km en Navarra, 11 km en Guipúzcoa y es fronterizo con Francia hasta su desembocadura en Fuenterrabía. La superficie de la cuenca es de $706 \mathrm{~km}^{2}$. En cabecera la pendiente es de $20 \mathrm{~m} / \mathrm{km}$, sin embargo en el resto del río la pendiente disminuye considerablemente hasta $5 \mathrm{~m} / \mathrm{km}$. El río tiene carácter torrencial con precipitación media anual de $2148 \mathrm{~mm}$ para el período 1940141 a 1982/83 (CHNE, 1986). El sustrato está básicamente compuesto de cantos rodados y guijarros. La actividad más importante a lo largo de su cuenca es agrícola-ganadera con cría, sobre todo, de ganado vacuno y porcino. La acti- vidad industrial de la cuenca es poco importante con núcleos aislados situados en Lesaca y Vera de Bidasoa, donde existe un conjunto de industrias (laminaciones, galvanotecnia, talleres, etc.) (DFN, 1976).

El río Oria es el más importante de Guipúzcoa. Su recorrido es de $62 \mathrm{~km}$, con una cuenca de $850 \mathrm{~km}^{2}$ que ocupa la mitad de Guipúzcoa. Las pendientes son elevadas en cabecera con valores de $50 \mathrm{~m} / \mathrm{km}$ disminuyendo considerablemente a $4-7 \mathrm{~m} / \mathrm{km}$ aguas abajo. El río tiene carácter torrencial con precipitación media anual en la zona de 1516 mm para el período 1940141 a 1982/83 (CHNE, 1986). Con respecto a los aportes contaminantes, se dispone de los datos publicados por la Diputación Foral de Guipúzcoa (1982) en los que se aprecia que la actividad industrial en esta cuenca es de gran importancia. La zona de BeasainTolosa es la que soporta mayor concentración de industrias (talleres, pinturas, carrocerías, química, galvanotecnia, fundiciones). Entre los contaminantes se encuentran materia orgánica, sólidos en suspensión, cianuros, Zn, Ni, $\mathrm{Cr}$, disolventes, aceites y grasas.

El Lea es un río pequeño con $22 \mathrm{~km}$ de curso y se halla situado en la provincia de Vizcaya. Su cuenca, de forma

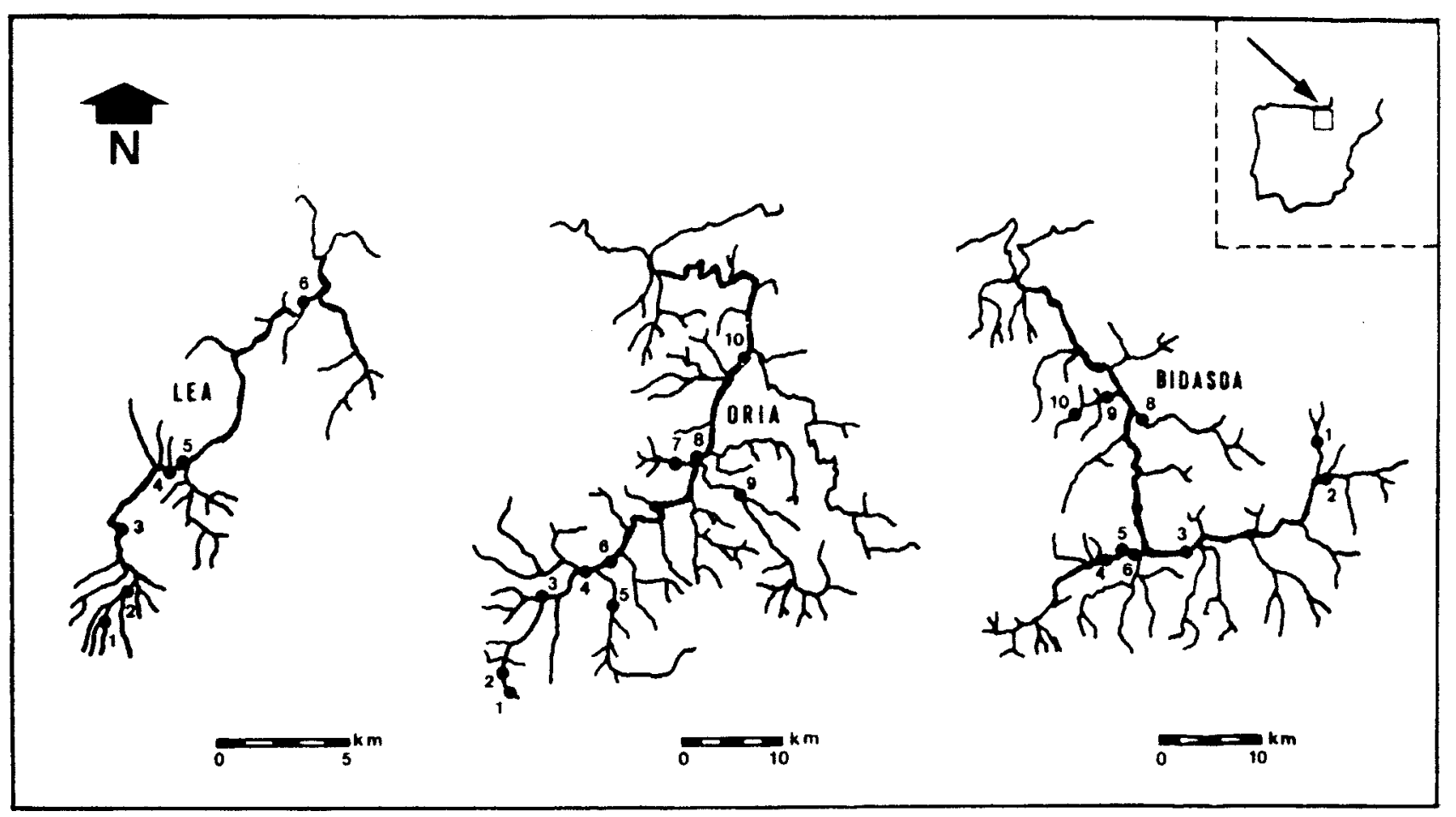

FIGURA 1.- Mapa de las cuencas de los nos Bidasoa, Oria y Lea.

FIGURE 1.- Geographic situation of sampling points in the basins of Bidasoa, Oria and Lea rivers. 
alargada, tiene un área de $80 \mathrm{~km}^{2}$. El cauce principal alcanza una altura máxima de $300 \mathrm{~m}$ en el límite sur y una pendiente de $13.6 \mathrm{~m} / \mathrm{km}$. ERASO (1983) ha realizado un amplio estudio morfómetrico. Este río de carácter torrencial presenta cargas contaminantes de origen urbano y agricola-ganadero, principalmente. La actividad industrial es poco importante con pequeñas empresas de transformación de madera (aserraderos y fábricas de muebles) y de conservas de pescado. Los vertidos son puntuales y en un $91 \%$ se hacen directamente al cauce principal (DFV, 1986).

\section{MÉTODOS}

Para la toma de muestras de agua se eligicron 12 puntos de muestreo en la cuenca del río Bidasoa (cinco de la cuenca principal), 10 en el Oria (tres en la cuenca principal) y 4 en el Lea. El muestreo se realizó durante un año (1987188) con periodicidad mensual.

Los parámetros físico-químicos determinados fueron temperatura, pH y conductividad (medidos in situ), alcalinidad, sílice. amoniaco, fósforo reactivo, fósforo total, nitrato, cloruro, sulfato, calcio, magnesio, sodio y potasio.

Las muestras de sedimento se tomaron en 10 puntos del río Bidasoa (tres en el cauce principal), 10 en el Oria (seis en el principal) y 6 en la cuenca del Lea. Se tomaron muestras superficiales $(3$ a $5 \mathrm{~cm}$ ). El sedimento se secó a $50-60{ }^{\circ} \mathrm{C}$, durante $24-48 \mathrm{~h}$, en estufa y posteriormente se homogeneizó. De la muestra, se hicieron tres partes, una para realizar el análisis granulométrico, otra para el análisis total $(<2 \mathrm{~mm})$ y una tercera para determinar la composición de la fracción fina $(<0.063 \mathrm{~mm})$. Las determinaciones realizadas fueron carbono orgánico (C org), carbonatos (como $\mathrm{CO}_{3} \mathrm{Ca}$ ), capacidad de cambio
(CEC), Na, K, Ca, Mg, Cr, Pb, Ni, Cu, Zn, Mn, Cd, Al, $\mathrm{Fe}$ y $\mathrm{Si}$.

Los métodos químicos utilizados para las muestas de agua se ajustaron básicamente a métodos conocidos (APHA. 1980) con modificaciones específicas para algunas determinaciones como amoniaco (MACKERETH et al., 1978) y cloruros, nitratos y sulfatos determinados por cromatografía iónica (HARRISON et al., 1985). Para el análisis de los sedimentos se siguieron básicamente normas para análisis de suelos (ASA-SSSA, 1982) ensayados y aplicados al tipo de muestra, utilizando para contraste una muestra patrón (NBS, 1988; RUIZ et al., 1991).

Como métodos estadísticos se utilizaron técnicas convencionales (CALVO, 1978) así como análisis factorial (MORRISON, 1976: CALVO, 1978) y dendrogramas de clasificación numérica (CUADRAS, 1981). Se utilizaron, así mismo, diagramas de Piper e índices de saturación de Langelier (DEGRÉMONT, 1989) en base a la caracterización de las aguas y el estudio de los equilibrios de especies iónicas (sistema carbonatos).

\section{RESULTADOS Y DISCUSIÓN}

Con los resultados medios de once parámetros medidos en las muestras de agua de las tres cuencas (Tablas 1, 2, 3) se han trazado los diagramas representativos de los constituyentes mayoritarios (DEGREMÓNT, 1989; RUIZ. 1990).

Las aguas del río Bidasoa son fundamentalmente bicarbonatadas cálcicas, con las excepciones de los puntos de muestreo 11 y 12 (próximos a desembocadura) en los que el carácter predominante es clorurado cálcico. La tabla 1 contiene, así mismo, los valores de $\mathrm{pH}$ de equilibrio y su dependencia con el $\mathrm{pH}$ real $(\Delta \mathrm{pH})$ que indican su equili-

TABLA 1.- Valores medios de los parámetros medidos en muestras de agua del río Bidasoa y valores de pH de equilibrio TABLE 1.- Average values of parameters and equitibrium $\mathrm{pH}$ in waters from Bidasoa rivcr.

\begin{tabular}{|c|c|c|c|c|c|c|c|c|c|c|c|c|c|c|c|}
\hline $\begin{array}{l}\text { Punto de } \\
\text { muestreo }\end{array}$ & $\begin{array}{r}\begin{array}{c}\text { Cloruros } \\
\mathrm{mg} \mathrm{Cl} /\end{array} \\
\end{array}$ & $\begin{array}{c}\text { Nitratos } \\
\mathrm{mg} \mathrm{N} 0311\end{array}$ & $\begin{array}{c}\text { Sulfatos } \\
\text { rng S0411 }\end{array}$ & $\begin{array}{l}\text { Alcal } \\
\text { meq } A\end{array}$ & $\begin{array}{c}\text { Sllice } \\
\text { rng Si/l }\end{array}$ & $\begin{array}{c}\mathrm{P} \text { react } \\
\mu \mathrm{P} A\end{array}$ & $\begin{array}{l}\text { P Total } \\
\mu g \mathrm{P} / 1\end{array}$ & $\begin{array}{c}\text { Calcio } \\
\text { rng Ca/l }\end{array}$ & $\begin{array}{r}\text { Magnesio } \\
\mathrm{mg} \mathrm{Mg/1}\end{array}$ & $\begin{array}{c}\text { Sodio } \\
\mathrm{mg} \mathrm{Na} / \mathrm{l}\end{array}$ & $\begin{array}{l}\text { Potasio } \\
\mathrm{mg} \mathrm{K} / \mathrm{I}\end{array}$ & pALC & C & $\mathrm{pH} \in \mathrm{q}$. & $\Delta \mathrm{pH}$ \\
\hline Cegama & 277.21 & 3.40 & 47.80 & 2.60 & 223 & 2389 & 4669 & 6157 & 439 & 16419 & 2.93 & 2.60 & 2.53 & 788 & 061 \\
\hline Mutiloa(*) & 3967 & 278 & 7184 & 3.26 & 2.47 & 48.84 & 83.13 & 72.75 & 8.40 & 24.30 & 286 & 250 & 2.48 & 768 & 0.71 \\
\hline Aya () & 16.18 & 3.54 & 22.30 & 254 & 111 & 79.39 & 13286 & 4850 & 302 & 1083 & 2.36 & 255 & 2.45 & 790 & 051 \\
\hline Ataun $\left({ }^{*}\right)$ & 466 & 267 & 1576 & 2.25 & 135 & 700 & 20.37 & 4342 & 2.24 & 485 & 089 & 265 & 249 & 814 & 0.20 \\
\hline Alegria & 2481 & 6.03 & 3797 & 3.16 & 188 & 30334 & 48511 & 5960 & 425 & 21.78 & 4.00 & 250 & 2.41 & 766 & 022 \\
\hline Albistur(*) & 720 & 431 & 2497 & 285 & 199 & 1523 & 33.98 & 5820 & 399 & 528 & 1.26 & 250 & 247 & 772 & 0.53 \\
\hline Betelu(*) & 1888 & 268 & 50.22 & 292 & 1.11 & 2153 & 5281 & 6609 & 5.98 & 1119 & 1.47 & 250 & 2.43 & 765 & 074 \\
\hline Lizarza(*) & 501 & 267 & 7052 & 289 & 129 & 790 & 1880 & 7390 & 8.33 & 3.47 & 086 & 250 & 248 & 768 & 081 \\
\hline Leiza(*) & 839 & 423 & 1145 & 2.01 & 1.48 & 85.80 & 146.48 & 4456 & 205 & 445 & 112 & 2.70 & 245 & 810 & 029 \\
\hline Lasarte & 2566 & 469 & 6190 & 243 & 175 & 7426 & 19453 & 6283 & 485 & 1770 & 247 & 255 & 240 & 770 & 030 \\
\hline
\end{tabular}


TABLA 2.- Valores medios de los parámetros medidos en muestras de agua del río Oria y valores del $\mathrm{pH}$ de equilibrio

TABLE 2.- Average values of parameters and equilibrium $\mathrm{pH}$ in waters from Ona river

\begin{tabular}{|c|c|c|c|c|c|c|c|c|c|c|c|c|c|c|c|}
\hline $\begin{array}{l}\begin{array}{l}\text { Punto de } \\
\text { muestreo }\end{array} \\
\end{array}$ & \begin{tabular}{|l|}
$\begin{array}{l}\text { Cloruros } \\
\mathrm{mg} \mathrm{Cl} / \mathrm{l}\end{array}$ \\
\end{tabular} & $\begin{array}{c}\text { Nitratos } \\
\text { mg NO3A }\end{array}$ & $\begin{array}{c}\text { Sulfatos } \\
\text { mg SO } 4 \lambda\end{array}$ & $\begin{array}{l}\text { Alcal. } \\
\text { meq } 1\end{array}$ & $\begin{array}{c}\text { Silice } \\
\text { mg Sill }\end{array}$ & $\begin{array}{l}\text { P react. } \\
\mu \mathrm{g} P / /\end{array}$ & $\begin{array}{l}\text { P Total } \\
\text { pg P/l }\end{array}$ & $\begin{array}{c}\text { Cakio } \\
\text { mg Cal }\end{array}$ & $\begin{array}{c}\text { Magnesio } \\
\text { mg Mgfl }\end{array}$ & $\begin{array}{c}\text { Sodio } \\
\text { mg N } 4\end{array}$ & $\begin{array}{l}\text { Potasio } \\
\mathrm{mg} \mathrm{K} / 1\end{array}$ & pAC & c & pHeq. & $\Delta \varphi \mathrm{H}$ \\
\hline Maya & 5.12 & 1.85 & 13.45 & 1.06 & 2.10 & 11.65 & 25.86 & 16.56 & 4.42 & 3.65 & 0.99 & 2.95 & 247 & 8.77 & -0.68 \\
\hline Azpilikueta & 5.88 & 1.26 & 6.87 & 1.23 & 2.00 & 5.37 & 22.97 & 16.68 & 5.75 & 4.00 & 0.69 & 2.90 & 2.43 & 8.68 & -0.63 \\
\hline Arizkun $\left({ }^{*}\right)$ & 5.98 & 1.70 & 8.83 & 1.37 & 2.11 & 20.34 & 48.86 & 18.04 & 5.86 & 3.87 & 0.99 & 2.85 & 243 & 8.63 & -0.60 \\
\hline Venta & 5.85 & 1.93 & 12.04 & 116 & 1.99 & 17.58 & 37.91 & 16.94 & 4.96 & 3.79 & 1.07 & 292 & 2.42 & 8.69 & -0.59 \\
\hline Arrayoz & 9.34 & 2.25 & 22.28 & 1.71 & 166 & 3040 & 53.10 & 30.56 & 5.29 & 572 & 1.46 & 2.75 & 2.40 & 8.30 & 0.20 \\
\hline Ezkurra $\left({ }^{\circ}\right)$ & 4.47 & 1.88 & 9.98 & 1.84 & 1.74 & 7.32 & 13.97 & 34.91 & 1.73 & 3.16 & 087 & 2.72 & 2.48 & 8.30 & 0.02 \\
\hline Saldias (") & 4.79 & 2.19 & 10.37 & 1.98 & 1.75 & 21.27 & 41.61 & 37.31 & 222 & 3.22 & 1.14 & 2.70 & 2.48 & 8.23 & 0.12 \\
\hline Elgorriaga (*) & 6.50 & 4.09 & 11.73 & 1.36 & 1.62 & 67.00 & 103.26 & 25.38 & 2.34 & 4.13 & 1.36 & 2.85 & 2.44 & 8.49 & +026 \\
\hline Sumbilla & 7.66 & 2.01 & 17.76 & 163 & 1.72 & 23.27 & 48.58 & 27.55 & 4.38 & 5.22 & 1.19 & 2.80 & 2.43 & 8.38 & -0.40 \\
\hline Etxalar ()$\left.^{*}\right)$ & 7.58 & 1.72 & 7.11 & 0.73 & 2.24 & 14.95 & 3732 & 11.01 & 2.46 & 5.44 & 0.95 & 315 & 2.42 & 9.12 & -1.41 \\
\hline Losaka & 47.88 & 2.96 & 13.06 & 1.33 & 2.92 & 47.67 & 90.97 & 42.90 & 2.45 & 6.08 & 137 & 2.90 & 244 & 8.29 & -0.44 \\
\hline Endara (*) & 8.07 & 2.96 & 5.85 & 0.39 & 3.14 & 3.67 & 23.89 & 6.85 & 1.24 & 5.42 & 0.84 & 3.42 & 2.41 & 958 & -2.06 \\
\hline
\end{tabular}

TABLA 3.- Valores medios de los parámetros medidos en muestras de agua del río Lea y valores de $\mathrm{pH}$ de equilibrio

TABLE 3.- Average values of parameters and equilibnum $\mathrm{pH}$ in waters from Lea nver.

\begin{tabular}{|c|c|c|c|c|c|c|c|c|c|c|c|c|c|c|c|}
\hline $\begin{array}{l}\text { Punto de } \\
\text { muestreo }\end{array}$ & $\begin{array}{l}\text { Cioruros } \\
\mathrm{mg} \mathrm{Cl} /\end{array}$ & $\begin{array}{c}\text { Nitratos } \\
\text { mg NO3A }\end{array}$ & $\begin{array}{c}\text { Sulfatos } \\
\mathrm{mg} \text { SO } 4 \AA\end{array}$ & $\begin{array}{l}\text { Alcai. } \\
\text { meq } A\end{array}$ & $\begin{array}{c}\text { Sílice } \\
\text { mg Si/l }\end{array}$ & $\begin{array}{l}\text { P react. } \\
\mathrm{pg} \mathrm{P} / 1\end{array}$ & $\begin{array}{l}\text { P Total } \\
\mu \mathrm{g} P / \mid\end{array}$ & $\begin{array}{l}\text { Cakio } \\
\text { mg Ca/l }\end{array}$ & $\begin{array}{c}\text { Magnesio } \\
\text { mg Mgfl }\end{array}$ & $\begin{array}{c}\text { Sodio } \\
\text { mg Na/n }\end{array}$ & $\begin{array}{l}\text { Potasio } \\
\mathrm{mg} \mathrm{K} / \mathrm{l}\end{array}$ & pALC & $c$ & pHeq. & $\mathrm{ApH}$ \\
\hline Guizaburuaga & 10.15 & 3.37 & 9.96 & 2.90 & 2.39 & 8.89 & 23.55 & 55.28 & 2.34 & 8.33 & 1.07 & 2.55 & 2.48 & 7.88 & 0.46 \\
\hline Aulestia & 12.04 & 3.10 & 17.29 & 2.39 & 2.60 & 15.54 & 30.93 & 46.96 & 3.32 & 8.08 & 2.10 & 2.65 & 2.48 & 807 & 0.11 \\
\hline Guerricaiz & 15.66 & 3.03 & 16.37 & 2.43 & 1.94 & 14.32 & 32.86 & 47.39 & 3.05 & 9.61 & 1.84 & 265 & 2.43 & 805 & 000 \\
\hline Oleta & 18.60 & 3.30 & 16.00 & 2.54 & 1.76 & 17.39 & 40.94 & 4981 & 307 & 10.90 & 2.01 & 2.60 & 2.42 & 792 & 0.15 \\
\hline
\end{tabular}

brio carbónico. Las aguas son en su mayoría débilmente agresivas, con las excepciones de los puntos de muestreo 5,6 y 7 (que están prácticamente en equilibrio) y 12 (fuertemente agresiva).

Las aguas del río Oria (Tabla 2) son mayoritariamente bicarbonatadas cálcicas y débilmente incrustantes en su totalidad. La muestra numero 1 próxima a Cegama tiene carácter clorurado sódico. La explotación de salmueras para el beneficio de cloruro sódico (DFG, 1982) justifican la composición anómala de esta muestra de agua.

Por último, el río Lea (Tabla 3) tiene aguas débilmente incrustantes y bicarbonatadas cálcicas.

Con los datos de las muestras de aguas de los tres ríos se ha realizado un tratamiento conjunto en base a establecer relaciones entre los distintos contituyentes químicos y diferentes puntos de muestreo. Para ello se han utilizado análisis factorial y dendrogramas de clasificación numérica.

El análisis factorial (método de componentes principales con rotación de ejes) para los distintos constituyentes condujo a la obtención de seis componentes, con una varianza global explicada del $95.42 \%$.

Los dos componentes que explican el mayor porcentaje de varianza se correlacionan negativamente con los nutrientes (amoniaco, nitratos, fósforo reactivo, fósforo total y potasio) y positivamente con sodio, potasio, cloruros y conductividad, respectivamente.

Los otros dos componentes en importancia están asociados mayormente a alcalinidad y calcio y con los sulfatos y el magnesio de forma positiva, respectivamente.

El trazado de dendrogramas de clasificación de variables lleva a resultados análogos (Fig. 2), apreciándose las siguientes agrupaciones:

* Los micronutrientes fósforo reactivo, fósforo total, amoniaco, nitratos y potasio deben atribuirse a un fenómeno de contaminación probablemente por fertilizantes.

* Conductividad, cloruros y sodio.

* Alcalinidad, calcio, sulfatos y magnesio, asociados probablemente a componentes del terreno ricos en bicarbonato cálcico y sulfato magnésico.

* Sílice y pH no se relacionarían, si bien del mapa de posición (no incluido) se deduce que estarían relacionados negativamente entre si.

Se han trazado de forma similar los mapas de posición de observaciones (puntos de muestreo). De las veintisiete muestras de agua, las muestras 1-4 pertenecen al Lea, 5-16 al Bidasoa y 17-27 al Oria.

En los mapas de posición de los componentes se observa una separación de las muestras del río Oria respecto a 
TITULO DEL ESTUdiO : COMPOSICION DE LAS AGUAS.

OENDOGRAMA DE CLASIFICACION DE VARIABLES.

BASADO EN DISTANCIAS $1-r$ ( $r=$ coeficiente de correlación) CALCULADO POR EL METODO DE MEDIAS PONDERADAS (UPGMA)

COEFICIENTE DE CORRELACION COFENETICA, RCof . $=0.907$

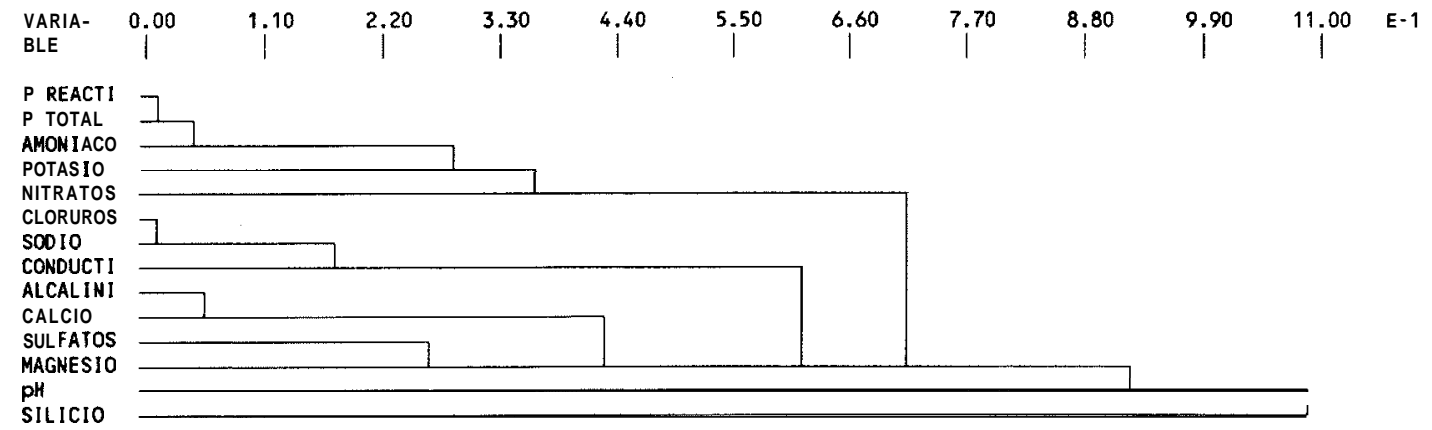

FIGURA 2.- Dendrograma de clasificación de todas las variables.

FIGURE 2.- Cluster analysis. Dendrograrn of classifcation of variables.

las del Lea y Bidasoa (RUIZ, 1990). Especialmente contaminada en contituyentes orgánicos (fósforo, amoníaco y nitratos) estaría la muestra número 21 (correspondiente a Alegría de Oria). La posible contaminación agraria estaría reforzada en este punto por contaminación de tipo urbano e industrial (detergentes, nitrógeno orgánico). Los puntos 17 y 18 ricos en sodio y cloruros, están situados en la cabecera del río donde existe una zona de afloramiento de Keuper rico en estas sales.

Estas diferencias de las muestras del río Oria se manifiestan tambien respecto a su mayor contenido en bicarbonato cálcico y sulfato magnésico.

El dendrograma de clasificación para los diferentes puntos de muestreo (Fig. 3) recoge esta información, remarcando la composición anómala de la muestra 21 ya comentada.

Los sedimentos en contacto con estas aguas presentan composiciones molares medias en cationes mayoritarios que se recogen en gráficas comparables a los diagramas de Piper (Fig. 4, 5, 6).

Los sedimentos del río Bidasoa son así fundamentalmente pobres en calcio y presentan contenidos molares sensiblemente iguales en $\mathrm{Mg}$ y $\mathrm{Na}+\mathrm{K}$. La carencia de calcio resulta más acentuada en la muestra total. El río Oria presenta, por otra parte, sedimentos ricos en calcio, mientras que el río Lea resulta rico en $\mathrm{Na}+\mathrm{K}$ en la muestra de sedimentos de tamaño $<0.063 \mathrm{~mm}$ y relativamente rico en calcio en la muestra total.

Con un criterio semejante se han calculado los porcen-

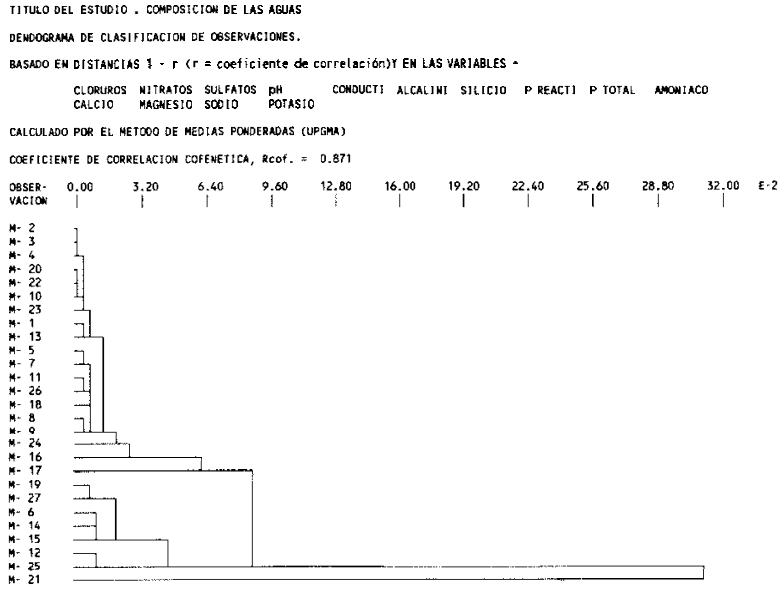

FIGURA 3.- Dendrograma de clasificación de observaciones. FIGURE 3.- Cluster analysis. Dendrogram of classifcation of samples.
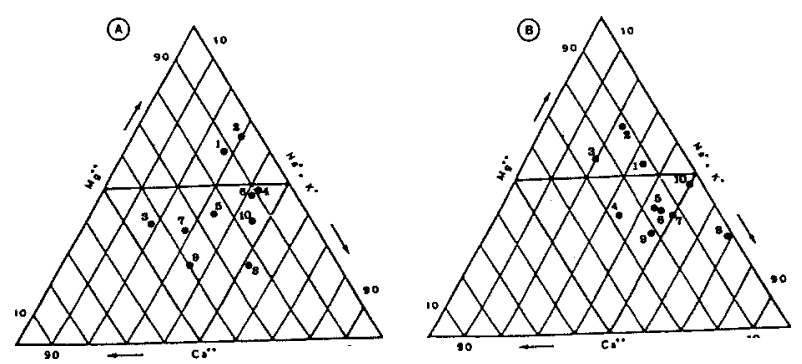

FIGURA 4.- Composiciones medias en cationes mayoritarios de sedimentos del no Bidasoa: a) muestra $<0.063 \mathrm{~mm}$. b) muestra total. FIGURE 4,- Unitary mean compositions of major cationic constituens of sediments from Bidasoa river: a) sample $<0.063 \mathrm{~mm}$. b) total sample. 

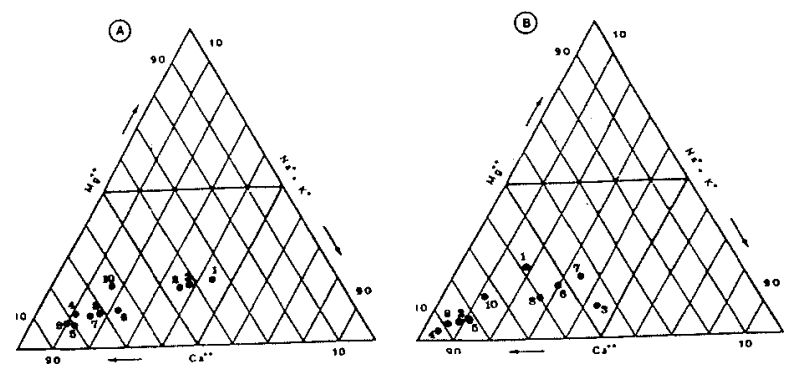

FIGURA 5. Composiciones medias en cationes mayoritarios de sedimentos del río Oria: a) muestra $<0.063 \mathrm{~mm}$. b) muestra total.

FIGURE 5.- Unitary mean compositions of major cationic constituens of sediments from Oria river: a) sample $<0.063 \mathrm{~mm}$. b) total sample.
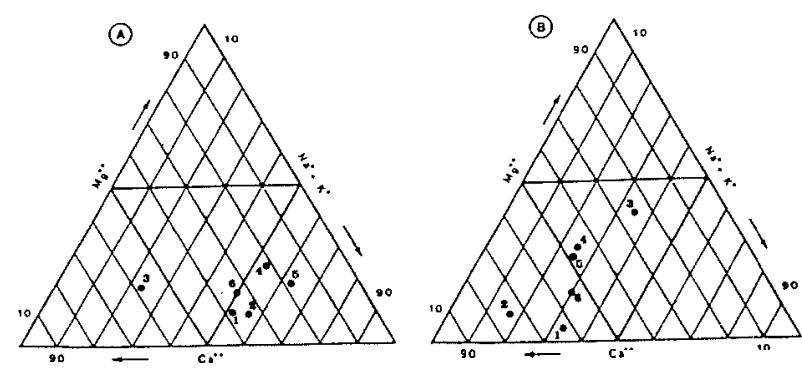

FIGURA 6. Composiciones medias en cationes mayoritarios de sedimentos del río Lea: a) muestra $<0.063 \mathrm{~mm}$. b) muestra total.

FIGURE 6.- Unitary mean compositions of major cationic constituens of sediments from Lea river: a) sample $<0.063 \mathrm{~mm}$. b) total sample.

tajes medios molares de carbonatos, aluminio y silicio de las muestras de sedimentos de los tres ríos, que se recogen en la Tabla 4. La observación de estos datos indica que:

1) Los sedimentos del río Oria son los más ricos en carbonatos, mientras que los del río Bidasoa son los más pobres (en especial en la muestra total). Resulta extraño el alto contenido medio en carbonatos de las muestras $<0.063 \mathrm{~mm}$ del río Bidasoa, con respecto a las totales y más teniendo en cuenta el caracter débilmente agresivo de las aguas de dicho río.
2) Las muestras totales presentan mayores contenidos medios en silicio y menores en aluminio que las $<0.063$ $\mathrm{mm}$ (salvo el río Oria en que los contenidos son sensiblemente iguales). Además, se aprecia para los ríos Bidasoa y Lea un aumento considerable de la relación $\mathrm{Al} / \mathrm{Si}$ en la muestra $<0.063 \mathrm{~mm}$. El río Oria presenta valores sensiblemente iguales en ambas muestras. Este aumento parece atribuible a la presencia de mayor contenido en arcillas en las fracciones $<0.063 \mathrm{~mm}$ de aquellos ríos.

3) Las relaciones $\mathrm{Al} / \mathrm{Si}$ existentes en minerales comunes (AMORÓS, 1951) son:

$\begin{array}{lr} & \mathrm{Al} / \mathrm{Si} \\ \text { Sílice } & 0.00 \\ \text { Feldespatos (Albita, Ortociasa) } & 0.25 \\ \text { Minerales de la arcilla: } & \\ \quad \text { Montmorillonita } & 0.33 \\ \quad \text { Caolinita } & 0.50\end{array}$

Ello sugiere la existencia de sílice libre en las muestras totales, así como la probable presencia de minerales de la arcilla de tres capas (montmorillonita) pero una escasa proporción de los de dos capas (caolinita).

En consecuencia con todo lo anterior debe sugerirse que, a causa del carácter torrencial de los ríos estudiados y del tiempo presumiblemente corto de contacto entre agua y sedimentos, no parece haberse llegado a situaciones de equilibrio. Por ello, parece mucho más lógico asignar la composición de las aguas a los constituyentes solubilizados durante su transcurso y la composición media de los sedimentos al material residual arrastrado, procedente de la disgregación de las rocas con las que el agua estuvo previamente en contacto. Otro aporte, que puede ser importante para los sedimentos en algunos puntos es, con todo, la existencia de vertidos de carácter agropecuario, urbano e industrial, que pueden influir en los contenidos de ciertos constituyentes, en especial en materia orgánica y microconstituyentes metálicos.

TABLA 4.- Porcentajes medios molares de carbonatos, aluminio y silicio en las muestras de sedimento de los ríos Bidasoa, Oria y Lea TABLE 4.- Mean molecular percentages of carbonate, aluminium and silicium in sediments from Bidasoa, Oria and Lea rivers.

\begin{tabular}{|c|c|c|c|c|c|c|c|c|}
\hline & \multicolumn{3}{|c|}{$<63 \mu \mathrm{m}$} & \multicolumn{5}{|c|}{ TOTAL } \\
\hline & $\mathrm{CO3}$ & AL & $\mathrm{Si}$ & $\begin{array}{c}\text { Relación } \\
\text { Al/si }\end{array}$ & $\cos$ & Al & $\mathrm{Si}$ & $\begin{array}{c}\text { Relación } \\
\text { Al/si }\end{array}$ \\
\hline BIDASOA & 5,98 & 24,14 & 69.88 & 0,345 & 0.91 & 16,26 & 82.83 & 0,196 \\
\hline ORIA & 9,48 & 17,82 & 72,70 & 0,245 & 11,01 & 19,67 & 69.32 & 0,284 \\
\hline LEA & 6,60 & 27,53 & 65.87 & 0,418 & 5,12 & 12,00 & 82,88 & 0,145 \\
\hline
\end{tabular}




\section{BIBLIOGRAFÍA}

APHA, 1980. Standard Methods for the examination of water and waste water. 15 th. Edition.

AMORóS, J.L., 1951. Cristaloquímica, Cap. 5-6. Consejo Superior de Investigaciones Científicas. Monografías de Ciencias Modernas, pp 37.

ASA-SSSA, 1982. Methods of Soil Analysis. Part 2Chemical and Microbiological Properties. 2nd Edition. A.L. PAGE, R.H. MILLER \& D.R. KEENEY (ed). ASASSSA. Madison, Wisconsin, USA.

CALVO, F., 1978. Estadistica Aplicada. Cap. 13. Ediciones Deusto S.A., Bilbao.

CASEY, H., 1976. The changing chemical composition of chalk in streams and rivers. Freshwater Biol. Assoc. Annual Report: 28-35.

CASEY, H \& NEWTON P.V.R., 1973. The chemical composition and flow of the River From and its main tributaries. Freswater Biol. 3: 317-333.

CHNE, 1986. Recursos hidraúlicos de las cuencas del Norte de España. Sintesis. Estudios previos de planificación hidrológica. Confederación Hidrográfica del Norte de España.

CUADRAS, C.M., 1981. Métodos de análisis multivariante. Editorial Universitaria de Barcelona. Barcelona.

DEGREMONT, 1989. Memento Technique de L'eau. Neuviéme édition. Tome 1, 2.

DFG, 1982. Diputación Foral de Guipúzcoa. Estudio del seguimiento de la contaminación del río Oria.

DFN, 1976. Diputación Foral de Navarra. Estudio hidrológico de la cuenca del Bidasoa.

DFV, 1986. Diputación Foral de Vizcaya. Plan Director de Saneamiento de la cuenca del río Lea. Sener.
EINSTEIN, H.A. \& KRONE, R.B. 1962. Experiments to determine modes of cohesive sediment transport in salt water. J.Geophys. Res. 67: 1451-1461.

ERASO, A., 1983. Estudio morfométrico de las cuencas de los ríos Butrón, Oca, Lea y Artibai (Vizcaya). Lurralde: investigación y espacio $6: 35-80$.

FORSTNER, U. \& PROSSI, F., 1978. Heavy metal pollution in freshwater ecosystems. Biological aspects of freshwater pollution. pp 129-161. Commision of the European Communities Ed. Pergamon Press.

HARRISON, K; BECKMAN, W.C. Jr; YATES, T.; CARR, C.D., 1985. Rapid, costeffective ion chromatography. American Laboratory. May 1985.

MACKERETH, F.J.H.; HERON, J. \& TALLING. J.F., 1978. Water analysis: some revised methods for limnologists. Freshwater Biological Association. Scientific Publication, 36.

MORRISON, D.F., 1976. Multivariate statistical methods. Cap. 5, 2nd ed. McGraw-Hill, New York, USA.

NBS, 1988. Standard Reference Material 2704. Buffalo River Sediment. Office of Standard References Materials. Chemistry Building, National Bureau of Standards, Galthersburtg, MD 20899, USA.

RUIZ, E., 1990. Identificación, origen y factores fisicoquímicos que condicionan la contaminación por elementos metálicos de sedimentos de ríos. Tesis doctoral. UPV.

RUIZ, E.; ECHEANDIA, A. \& ROMERO, F., 1991. Microanalytical determination of metallic constituents of river sediments. Fresenius J. Anal. Chem, 340: 223-229.

SALOMONS, W. \& FORSTNER, U., 1984. Metals in the hydrocycle. Springer Verlag, New York, USA. 